\section{Effects of focusing strategy on speeded classification with grouping, filtering,} and condensation tasks*

\author{
RICHARD L. GOTTWALD† and W. R. GARNER \\ Yale University, New Haven, Connecticut 06510
}

Speeded classification was studied with three tasks varying in the way in which extra stimuli are provided for a single class: grouping, with additional levels on a single relevant dimension; filtering, with additional levels on an irrelevant dimension; and condensation, with additional levels on a second relevant dimension. (Both relevant dimensions must be processed for correct classification.) For the dimensions used (color and form), filtering was easiest, followed by grouping and condensation. In the latter two cases, asymmetric classifications, in which one class had a single member, eliminated the difficulty of classification. The presumed mechanism is focusing, in which the single stimulus is seen as a positive set and all other stimuli as negative.

In simple choice reaction time experiments, each response is paired with a single stimulus. If more than one stimulus is appropriate to each response, the task becomes one of speeded classification. The number of stimuli assigned to a particular response can be increased in three basically different ways, and these lead to logically different information processing tasks.

\section{TASKS}

\section{Grouping}

The number of levels on a single relevant dimension is increased, with more than one level being assigned to a single response category (e.g., one response is assigned to red and yellow circles and a second to blue and green circles). This task requires that $S$ group two or more levels into a single category, and Keele (1970) observed an increase in classification time when he required grouping for either a color or a form dimension.

\section{Filtering}

The number of levels on a relevant dimension is the same as the number of response categories, but additional stimuli per category are provided by having two or more levels on an irrelevant dimension. Thus, the stimuli vary in two dimensions, but just one is relevant to the classification (e.g., one response is assigned to red circles and red squares and a second to blue

\footnotetext{
* This research was supported by Grant MH 14229 from the National Institute of Mental Health to Yale University. The authors wish to thank Elinor Garner for her help in data collection and tabulation in Experiment 2.

+ Now at Indiana University. Requests for reprints should be sent to Richard $L$. Gottwald, Department of Psychology, Indiana University at South Bend, 1825 Northside Boulevard, South Bend, Indiana 46615 .
}

circles and blue squares). Posner (1964) called this task filtering because it requires $S$ to filter out one dimension in order to classify stimuli properly.

\section{Condensation}

The third way to increase number of stimuli per response category is to use a second relevant dimension such that both dimensions must be evaluated before a correct response assignment can be made (e.g., one response is assigned to red circles and blue squares and a second to blue circles and red squares). Posner (1964) called this task condensation and argued that it would require a greater classification time than filtering tasks, a result which has been found in several studies (Fitts \& Biederman, 1965; Morin, Forrin, \& Archer, 1961).

\section{TASK DEMANDS AND}

SUBJECT STRATEGIES

The three tasks have been described in terms of the logical requirements of the tasks. However, $S$ is not guaranteed to perform in any particular manner, and how he does perform is an experimental question. For example, in filtering tasks, Garner and Felfoldy (1970) demonstrated that filtering actually occurs only for certain pairs of stimulus dimensions, termed separable, while it does not occur for other pairs of dimensions, termed integral. So, for integral dimensions, the term "filtering" is really a misnomer if used to refer to what $S$ does rather than to the logical the part of $S$ may influence information processing in these several tasks.

\section{Focusing}

One strategy $\mathbf{S}$ may use is to focus attention on just one set of stimuli, nature of the task. Other strategies on the positive set. Gottwald (1971) found that performance in a concept attainment task was affected by whether the response labels were neutral (alpha vs beta) or positive/negative (alpha vs not-alpha). When positive/negative labels were used, Ss focused on the properties of the positive set of stimuli. This focus facilitated performance if the alternative sets of stimuli were logically identical. If the two sets were logically different, then focusing was facilitative or detrimental, depending on which set was the positive one.

If a focusing strategy were used in a speeded classification task, the task would become one of deciding whether or not a stimulus belonged in the positive set rather than one of deciding in which of two sets a stimulus belonged. The result of such a strategy would be that increasing the number of stimuli in the negative set would not affect speed. The evidence that Ss use a focusing strategy is somewhat mixed. Hawkins and Hosking (1969) obtained results consistent with a focusing strategy, but LaBerge and Tweedy (1964) and Krueger (1970) obtained results inconsistent with such a strategy. However, Hawkins and Hosking presented evidence that a focusing strategy is used when the two stimulus sets differ greatly in number of stimuli and is especially used when there is only one stimulus in one of the sets.

\section{Purpose}

The present experiments are designed to explore aspects of grouping, filtering, and condensation tasks and, in particular, to determine the effectiveness of a focusing strategy for the grouping and condensation tasks.

\section{EXPERIMENT 1}

Experiment 1 used a card-sorting procedure. It provides a comparison between a filtering and a grouping task for the dimensions of color and form. Previous research has already shown that both the filtering task (Morin, Forrin, \& Archer, 1961; Fitts \& Biederman, 1965) and the grouping task (Keele, 1970) are performed faster than the condensation task. Performance is also examined with an asymmetric grouping task and an asymmetric condensation task, both of which should make a focusing strategy advantageous.

\section{Conditions}

The stimuli varied in color and form. The colors were red, yellow, blue, and green. In Munsell notation, the colors were $5 R / 4 / 14,5 Y / 8.5 / 14$, $5 \mathrm{~PB} / 3 / 10$, and $2.5 \mathrm{G} / 5 / 8$. The forms were circle, square, and equilateral 
Table 1

Sorting Times for Each Condition in Experiment 1

Relevant Attribute

\begin{tabular}{|c|c|c|c|c|}
\hline \multirow[b]{2}{*}{ Task } & & \\
\hline & & Color & Form & Mean \\
\hline Control & $010^{*}$ & $.380 t$ & .399 & .390 \\
\hline Filtering & \begin{tabular}{l|l}
0 & 0 \\
0 & 0
\end{tabular} & .386 & .406 & .396 \\
\hline Groupins & 00100 & .437 & - & - \\
\hline Asymmetric Grouping & 0100 & .382 & - & - \\
\hline Asymmetric Condensation & \begin{tabular}{l|l}
0 & 0 \\
0 & 0
\end{tabular} & 一 & - & .398 \\
\hline
\end{tabular}

*These diagrams illustrate the logical dimensional structure of the classifications, vertical and horizontal being the two dimensions and the partitioning being as indicated. tMean sorting times per stimulus, in seconds.

triangle with areas of $2.83,2.56$, and $1.89 \mathrm{~cm}^{2}$, respectively, chosen to give approximately equal appearing areas.

Five different tasks were used, and these are listed and shown schematically in Table 1.

The first task was a control. Within a deck of 32 cards, there were two stimuli which differed on one dimension, and $S$ had to sort the cards into two piles. Within the control task either color or form was relevant, and within each of these conditions, two different pairs of stimuli were sampled. Specifically, when color was relevant, one deck required sorting red vs blue circles and the other red vs yellow squares. When form was relevant, one deck required sorting yellow circles vs triangles and the other blue squares vs triangles.

The second task was a filtering task in which there were four different stimuli varying in two dichotomous dimensions within a deck of 32 cards. $S$ had to sort the cards into two piles on the basis of a single relevant dimension. The filtering task was also done with either color or form relevant, and two different decks were used for each relevant dimension. When color was relevant, one deck required sorting red vs blue circles and squares and the other, red vs yellow triangles and squares. When form was relevant, one deck required sorting red and blue circles vs squares and the other, red and yellow triangles vs squares.

The third task was a grouping task. Within a deck of 32 cards, there were four stimuli which varied along one dimension, and $S$ had to sort two stimuli into each of two piles. In this task, the stimuli were circles varying in color (red, yellow, blue, and green). Three different decks were used corresponding to the three ways in which two pairs of stimuli might be selected from a set of four.

The fourth task was an asymmetric grouping task. Within a deck of 32 cards there were three stimuli which varied along one dimension, and $S$ had to sort one stimulus into one pile and two stimuli into the other. In this task, the stimuli were circles varying in color (red, yellow, blue). Three conditions were used corresponding to the three ways in which three stimuli may be divided into groups of one and two.

The fifth task was an asymmetric condensation task in which there were four different stimuli varying on two dichotomous dimensions within a deck of 30 cards. $S$ had to sort one stimulus into one pile and three into the other. Since color and form were both relevant, there was only one condition within this task, and again two different decks were used. One deck required sorting blue circles vs yellow circles, blue squares, and yellow squares. The other deck required sorting yellow squares vs red squares, yellow triangles, and red triangles.

Within each deck, the sizes of the stimulus sets assigned to each response were equal, and within each set, each of the different stimuli occurred equally often.

\section{Procedure}

A total of 16 Ss was drawn from the psychology $S$ pool at Yale. Time per $S$ was approximately $1 \frac{1}{2} \mathrm{~h}$.

All measures were obtained from the sorting of decks of stimulus cards (white, $8.9 \mathrm{~cm}$ high $\times 6.3 \mathrm{~cm}$ wide) into two piles. Ss were instructed to sort as fast as possible but without making any errors. With each deck to be sorted, examples of the stimuli which belonged in each pile were laid out in front of $S$. $S$ held the deck in either hand face up and sorted the cards into two piles corresponding to the examples which were left in view.

The $S$ was given 16 practice trials (one on each of the 16 different decks) and then 64 test trials (four on each of the 16 different decks). After each deck had been sorted, time to the nearest $0.01 \mathrm{sec}$ was noted from an electronic counter operated by $\mathrm{E}$, and any errors were recorded. While $\mathbf{S}$ was sorting, E checked errors, recorded scores, and shuffled the next deck of cards to be used. S was not informed about either his time or error scores.

A Latin square was formed with 16 Ss, 16 decks, and 16 orders. This Latin square determined the order of the first 16 trials for $S$. Then this order was reversed for the next 16 trials, reversed again for the next 16 , etc. $S$ was given a 10 -min rest after the third block of 16 trials.

\section{Results}

The primary measure was sorting speed, in terms of time per card. Errors were few, averaging 0.22 per deck, and were evenly distributed across conditions, so no detailed analysis of errors was carried out. There were no large differences between the different decks used within each condition, so the data from the different decks were pooled. Table 1 shows mean sorting speeds for the various conditions. Unless otherwise noted, statistical analyses involved Ss by Conditions analyses of variance between pairs of conditions, and statements of significance are for $\mathrm{p}<.01$.

The filtering task was slower than the control task, though the difference was small, and when color was relevant, the difference was barely significant $(p<.05)$. The grouping task was considerably slower than the filtering task. Thus, for the dimensions used in the present study, the relative order of task difficulty is quite clear.

The asymmetric grouping task was much faster than the grouping task and was not different from the control task when color was relevant. This result indicates that $S s$ were using a focusing strategy and benefiting from it. Informal questioning at the end of the experimental sessions established that all Ss used a focusing strategy in the two asymmetric tasks. The Ss reported that they committed only one set of stimuli to memory and then sorted on a "yes-no" basis.

The sorting speed in the asymmetric condensation task also indicates that Ss were using a focusing strategy. Speed in this task was much faster than the speed in the grouping task, while Keele (1970) found that a symmetric condensation task was slower than a grouping task. The asymmetric condensation task was slower than the control when color was relevant but no different from the control when form was relevant. Since both form and color must be evaluated in the asymmetric condensation task and sorting speed was the same as that for form alone in the control task, it appears that both dimensions are being evaluated simultaneously. 
EXPERIMENT 2

The second experiment also used a card-sorting procedure. It was concerned with increasing the number of levels on both the irrelevant and the relevant stimulus dimensions in a filtering task. It also had one condition designed to allow both filtering and focusing to occur in a single task. And it had a condensation task.

\section{Conditions}

Specifically, six different conditions were used, and these are listed and shown schematically in Table 2.

The first task was filtering, which was identical to the filtering task used in Experiment 1.

The second task was filtering (3), which was similar to the filtering task, except that three levels of the irrelevant dimension were used rather than two.

The third task was 3-category filtering, which was similar to the filtering task, except that three levels of the relevant dimension were used and the cards were sorted into three piles.

The fourth task was 3-category filtering $(3,2)$, which was similar to the 3-category filtering task, except that three levels of the irrelevant dimension were used, but only two levels occurred in each category.

The fifth task was disjunctive filtering, in which there were four different stimuli within a deck, and two stimuli were sorted into each of two categories. The stimuli selected were such that stimuli within a category had one level of a dimension in common, but stimuli in different categories had no levels in common (e.g., blue triangles and blue squares vs red circles and yellow circles).

The sixth task was condensation. This task used the same stimulus sets as the 3-category filtering $(3,2)$, but only two responses were used. The responses were assigned so that two dimensions had to be used to classify a stimulus.

Every task was done with both color and form relevant, except for the disjunctive filtering and the condensation tasks. In the disjunctive filtering task, either dimension could have been used for classification, and in the condensation task, both dimensions had to be used for classification. The stimuli were selected from a set of three colors (red, yellow, blue) $x$ three forms (circle, square, triangle), and two different decks were sampled for each condition.

\section{Procedure}

A total of 12 Ss was drawn from a summer $S$ pool at Yale. The Ss were graduate and undergraduate students

Table 2

Sorting Times for Each Condition in Experiment 2*

\begin{tabular}{|c|c|c|c|c|c|}
\hline \multirow[b]{2}{*}{ Task } & & & \multicolumn{3}{|c|}{ Relevant Attribute } \\
\hline & & & Color & Form & Mean \\
\hline Filtering & $\begin{array}{l}0 \\
0\end{array}$ & $\begin{array}{l}0 \\
0\end{array}$ & .369 & .385 & .377 \\
\hline Filtering (3) & $\begin{array}{l}0 \\
0 \\
0\end{array}$ & $\begin{array}{l}0 \\
0 \\
0\end{array}$ & .366 & .383 & .374 \\
\hline 3-Category Filtering & & \begin{tabular}{l|l}
0 & 0 \\
0 & 0
\end{tabular} & .423 & .461 & .442 \\
\hline 3-Category Filtering $(3,2)$ & $\begin{array}{l}\mathbf{o} \\
\mathbf{0}\end{array}$ & \begin{tabular}{l|l}
0 & \\
& 0 \\
0 & 0
\end{tabular} & .427 & 462 & .444 \\
\hline Disjunctive Filtering & $\begin{array}{l}0 \\
0\end{array}$ & 0 o & 一 & - & .373 \\
\hline Condensation & $\begin{array}{l}\mathbf{0} \\
\mathbf{0}\end{array}$ & & - & - & .506 \\
\hline
\end{tabular}

*Tabular meanings the same as in Table 1

who were paid to participate.

The procedure was the same as that used in Experiment 1, except that each deck was sorted only three times (one practice and two test trials). In conditions in which there were four different stimuli, each deck contained 32 cards, and in conditions in which there were six different stimuli, each deck contained 30 cards.

\section{Results}

The mean sorting speeds for the various conditions are shown in Table 2. The number of errors was even lower than in Experiment 1 and averaged 0.09 per deck. Again, there were no large differences between the different decks used within each condition, and the data from the different decks were pooled.

The sorting speeds for the filtering task are slightly faster than the speeds obtained in Experiment 1, but the difference is not significant. The filtering task is faster when color is relevant than when form is relevant, and the difference is the same order of magnitude as the difference found in Experiment 1. In the filtering (3) task, color is faster than form ( $<<.05$ ), but the sorting speeds are not different from those obtained in the filtering task for either relevant dimension. Both 3-category filtering tasks are slower than any of the other filtering tasks. Color is faster than form in both tasks, and the two tasks do not differ from each other.

The sorting speed for the disjunctive filtering task is not different from the speeds for either the filtering or filtering (3) tasks when color is relevant, but it is faster than both tasks when form is relevant $[p<.05$ for the comparison with filtering (3)]

The sorting time for the condensation task is slower than the times for any of the other tasks. It is of some interest to compare the condensation task with the 3-category $(3,2)$ task, since both tasks involve the same set of stimuli. The condensation task is slower than the 3-category $(3,2)$ task for either relevant dimension ( $p<.05$ for form). Thus, the decrement in performance produced by having to evaluate two dimensions is greater than the decrement produced by an increase in the number of categories. The condensation task is also slower than the grouping task in Experiment 1 ( $p<.02$, Mann-Whitney $U$ test), which is consistent with the results obtained by Keele (1970).

\section{DISCUSSION}

The results of the present study show that the three information processing tasks differ in difficulty. The filtering task was easiest, followed by the grouping and the condensation tasks.

\section{Filtering}

In the first experiment, there was a slight and barely significant slowing of sorting speed when either form or color was added as an irrelevant variable with two levels. However, the second experiment showed that no further increase in number of levels on the irrelevant variable made the task more difficult. Overall, then, there is very slight interference produced by these two dimensions on each other, and they would be classed as essentially separable dimensions as contrasted with integral dimensions (Garner \& Felfoldy, 1970). However, any pair of dimensions cannot be used for a filtering task and, in fact, show evidence of successful filtering on the part of the $S$. Garner and Felfoldy used value and chroma of Munsell 
chips in a card-sorting task identical to that used in the present experiments and obtained a $23 \%$ increase in sorting time when these dimensions were used as relevant and irrelevant in a filtering task. This interference effect is even greater than that obtained with grouping. Since base levels of discriminability are not the same in the different experiments, exact comparisons are not valid. What is clear, however, is that very good filtering was obtained for the dimensions of form and color, as used in these experiments. A filtering task, however, will not necessarily produce filtering.

The role of focusing cannot be great when there is little interference in the filtering task. Since preliminary results anticipated the result obtained in the filtering task, a simple focusing condition was not used to of fset the effects of a filtering task. That focusing can be used in such tasks, however, is shown by the fact that focusing and filtering were both used in the disjunctive filtering task of the second experiment. That condition requires, in effect, that the $S$ first select a relevant dimension, focus on one level of the relevant dimension, and then ignore an irrelevant dimension within the positive category. While such a task sounds complicated as described, in practice it amounts to saying, for example, that the stimuli should be sorted so that all red stimuli go in one pile and everything else goes in the other pile. In this form it is indeed an easy task.

\section{Grouping}

The grouping task was quite difficult compared to the control condition, showing a $15 \%$ increase in sorting time. However, this disadvantage is completely lost when three stimuli are used in the asymmetric grouping task, which allows focusing on the stimulus set having a single member. Thus, there is no disadvantage to having more than one stimulus in a set if that set can be used simply as a negation to a positive set with a single member in it.

\section{Condensation}

Clearly, the most difficult task of all is condensation, a task in which both dimensions must be processed in order for a correct response assignment to be made. However, here again the use of a category with a single member allows focusing, and sorting times, in that case, are essentially the same as if the task were simple choice reaction. In this case, however, the focusing must be done with respect to both dimensions, and the very good sorting speeds obtained imply that both dimensions are processed at once. Possibly, however, the stimuli are not processed in a dimensional sense at all, but rather become a unitary stimulus, a template.

At this time, only conjecture is possible about whether the condensation task would be easier or more difficult with stimulus dimensions which are more integral than those used here. It seems entirely possible that with integral dimensions, the stimuli would be processed in unitary stimulus form, and that integral dimensions might make the condensation task more like the somewhat easier grouping task.

\section{Relationship to}

Perceptual Classification

Imai and Garner (1968) carried out a study using the same kinds of stimuli as those used in the present experiments, but with a free-classification technique in which $\mathbf{S}$ is shown various subsets of stimuli and asked to form classes any way he likes. Presumably, such a task is affected primarily by perceptual factors, while the results from reaction time experiments are affected by both perceptual and performance factors. It is of interest to compare the results of these experiments.

The perceptual classifications are characterized as preserving the dimensional structure of the stimuli as much as possible. If $S$ is required to partition a set of stimuli varying in two dimensions, he selects one of the dimensions as relevant and completely partitions the stimuli along the levels of that dimension. Such subsets would produce a filtering task for reaction time. Thus, the free-classification procedure produces subsets which will give fast reaction times.
However, there are some differences between the tasks. In classification, if the dimension selected as relevant has three levels, then the stimuli are partitioned into three subsets, and the results from the present experiments show that reaction time is made much slower by the use of three response categories. Furthermore, the use of subsets of single stimuli does not ordinarily occur in free classification. Thus, the use of classifications which would encourage focusing on one of two categories does not occur, even though such stimulus sets provide very rapid reaction times. It is clear that the results of the free-classification experiments reflect the nature of the perceived structure of the entire set of stimuli rather than performance factors.

\section{REFERENCES}

FITTS, P. M., \& BIEDERMAN, I. S-R compatibility and information reduction. Journal of Experimental Psychology, $1965,69,408-412$.

GARNER, W. R., FELFOLDY, G, L Integrality of stimulus dimensions in various types of information processing. Cognitive Psycholngy, 1970, 1, 225-241.

GOTTWALD, $R$. L. Effects. of response labels in concept attainment. Journal of Experimental Psychology, 1971, in press. HAWKINS, H. L.. \& HOSKING, K Stimulus probability as a determinant of discrete choice reaction time. Journal of Experimental Psychology, 1969, 82, 435-440.

IMAI. S.. \& GARNER, W. R. Structure in perceptual classification. Psychonomic Monograph Supplements, 1968, $2(9$ Whole No. 25), 153-172.

KEELE, S. W. Effects of input and output modes on decision time. Joumal of Experimental Psychology, 1970, 85, 157-164.

KRUEGER, L. E. Effect of stimulus probability on two-choice reaction time. Joumal of Experimental Psychology. J970,84, 377-379.

LAB ERGE, D., \& TWEEDY, J. R. Presentation probability and choice time. Journal of Experimental Psychology. $1964,68,477-481$

MORIN, R. E., FORRIN, B.. \& ARCHER $W$. Information processing behavior: The role of irrelevant stimulus information. Journal of Experimental Psychology. $1961,61,89-96$

POSNER, M. I. Information reduction in the analysis of sequential tasks. Psycholocical Review, 1964, 71, 491-504.

(Received for publication July 30, 1971.) 